\title{
The Damped Oscillator: A Locally Convex Formulation
}

By

\author{
Daniel A. Dubin* and Mark A. Hennings**
}

\begin{abstract}
We formulate the quantum system of an oscillator driven by a quantum Wiener process, in the locally convex setting based on the rigged triple $\mathcal{S}(\mathbb{R}) \subset L^{2}(\mathbb{R}) \subset$ $\mathcal{S}^{\prime}(\mathbb{R})$. The generalized observables are taken to be the elements of $\mathcal{L}\left(\mathcal{S}(\mathbb{R}), \mathcal{S}^{\prime}(\mathbb{R})\right)$. Pulling the dynamics back to phase space by means of the inverse of Weyl quantization, we prove that the time translations semigroup is equicontinuous of class $C_{0}$. Moreover, it is differentiable, and its generator is an extension to $\mathcal{L}\left(\mathcal{S}(\mathbb{R}), \mathcal{S}^{\prime}(\mathbb{R})\right)$ of the known result for bounded operators.
\end{abstract}

\section{$\S 1$. Introduction}

The damped harmonic oscillator is perhaps the best known example of a continuous open dissipative quantum system. Indeed, it hardly seems possible that there are any open questions concerning this model, nor any mathematical formalism for it which is not yet well established. The authors shared this point of view until recently, when they came to consider the inclusion of the damped oscillator as a subsystem of a more complex model.

In that model, the details of which need not concern us here, it is important that the principal quantum operators (including the non-Hamiltonian generator of the dynamical semigroup) be continuous mappings. This is, of course, not possible in the Hilbert space setting, but can be achieved using the formalism based on the rigged Hilbert space $\mathcal{S}(\mathbb{R}) \subset L^{2}(\mathbb{R}) \subset \mathcal{S}^{\prime}(\mathbb{R})[1,2,3]$. In addition, it is appropriate in that model to consider as observables those mappings obtained by quantizing (in the sense of Weyl $[4,5,6]$ ) all tempered distributions

Communicated by T. Kawai. Received April 7, 2003.

2000 Mathematics Subject Classification(s): Primary 81-02; Secondary 46h.

* Department of Pure Mathematics, The Open University, Milton Keynes MK7 6AA, UK.

** Sidney Sussex College, Cambridge CB2 3HU, UK. 
on the phase space $\Pi=\mathbb{R}^{2}$, which turn out to be precisely the elements of $\mathcal{L}\left(\mathcal{S}(\mathbb{R}), \mathcal{S}^{\prime}(\mathbb{R})\right)$. Continuity of these mappings can also be accommodated in the formalism.

In considering the damped oscillator subsystem, we were not able find any treatment of the time translation semigroup acting on $\mathcal{L}\left(\mathcal{S}(\mathbb{R}), \mathcal{S}^{\prime}(\mathbb{R})\right)$, nor its differentiability and the continuity of its generator. It turns out to be a somewhat lengthy and involved task to work all of this out in detail, and that is the purpose of this paper. In view of the centrality of the damped oscillator as a model, it seems to us that these details ought to be on record.

For most applications of quantum mechanics, but not all, quantization is not an issue, and one simply begins with the operators as quantum observables. Surprisingly, perhaps, we found that quantization provides just the right technical mechanism by which the necessary locally convex continuity estimates for the oscillator dynamics can be shown. The way it works is this: the actions of the time translations and its generator, which are known for bounded operators (and some unbounded ones, see Alli and Sewell [7]), are first pulled back through the quantization map to act on tempered distributions in phase space. This is formal at this stage, since we do not have any a priori proof that these transferred actions are continuous. But in the usual way for distributions, by the use of duality we cause them to act on the test functions, where continuity may be shown. It is then possible to combine the continuity of duality and the quantization map to prove that the original operations have the required properties.

We have oversimplified our description here, since we must also dilate the original oscillator system to account for the 'external forces', which are reflected in the non-unitary nature of the time evolution. We choose to mediate the external influences by means of a quantum Wiener process (see Hudson and Parthasaraty [8]). This is a free Boson field whose 'independent variable' has the dimensions of a time. The coupling we use is that previously employed by Alli and Sewell [7] in their seminal treatment of the Dicke-Hepp-Lieb laser model, c.f. $[4,9]$. In the familiar way, the enlarged system is conservative, and the dynamics of the original system is obtained from it by projection (compression).

The principal results of this paper are that the dynamics for the open oscillator is given through a differentiable and locally equicontinuous one parameter semigroup of class $C_{0}$, with continuous generator. Background material on locally convex spaces and distributions will be assumed (any of the texts $[10,11,12]$ will suffice). Our conventions concerning semigroups is that of 
Yosida [13]. In the appendix we have included a list of the seminorm families used in the paper.

One of us (DAD) gratefully acknowledges a number of conversations with Geoffrey Sewell on this and related matters.

\section{$\S 2 . \quad$ The Oscillator System}

As noted above, the kinematical description of the oscillator will be based on the Gel'fand rigged Hilbert space $\mathcal{S}(\mathbb{R}) \subset L^{2}(\mathbb{R}) \subset \mathcal{S}^{\prime}(\mathbb{R})$, where, in a standard notation, $\mathcal{S}\left(\mathbb{R}^{n}\right)$ is the test function space of infinitely differentiable functions on $\mathbb{R}^{n}$ decreasing more rapidly at infinity than any polynomial, and its dual, $\mathcal{S}^{\prime}\left(\mathbb{R}^{n}\right)$, is the space of tempered distributions.

$\mathcal{S}(\mathbb{R})$ will always carry its usual Frèchet topology, $\mathcal{S}^{\prime}(\mathbb{R})$ its strong dual topology. We shall also need the space $\mathcal{L}\left(\mathcal{S}(\mathbb{R}), \mathcal{S}^{\prime}(\mathbb{R})\right.$ ), whose elements (or at least the symmetric ones) are taken to be observables in a generalized sense. These include all the familiar observable operators on $L^{2}(\mathbb{R})$, but also mappings too singular to be operators. The space $\mathcal{L}\left(\mathcal{S}(\mathbb{R}), \mathcal{S}^{\prime}(\mathbb{R})\right)$ is equipped with its usual topology of uniform convergence on bounded subsets. With respect to this topology it is reflexive, and its strong dual may be identified with $\mathcal{S}\left(\mathbb{R}^{2}\right) \cong$ $\mathcal{S}(\mathbb{R}) \widehat{\otimes} \mathcal{S}(\mathbb{R})$ through

$$
\llbracket f \otimes g, X \rrbracket=\llbracket X g, f \rrbracket, \quad f, g \in \mathcal{S}(\mathbb{R}), X \in \mathcal{L}\left(\mathcal{S}(\mathbb{R}), \mathcal{S}^{\prime}(\mathbb{R})\right) .
$$

Note that we shall use $\llbracket \cdot, \cdot \rrbracket$ as a general duality symbol in the same way that $\langle\cdot, \cdot\rangle$ is a general inner product (complex conjugation on the left variable). The symbol $\widehat{\otimes}$ indicates completion of the tensor product in the projective tensor product topology. We note that as $\mathcal{S}(\mathbb{R})$ and its dual are reflexive, $\mathcal{L}\left(\mathcal{S}(\mathbb{R}), \mathcal{S}^{\prime}(\mathbb{R})\right)$ is linearly isomorphic and topologically homeomorphic to $\mathcal{S}^{\prime}(\mathbb{R}) \widehat{\otimes} \mathcal{S}^{\prime}(\mathbb{R})=\mathcal{S}^{\prime}\left(\mathbb{R}^{2}\right)$.

With our choice of rigged triple, we are working in the locally convex form of the Schrödinger representation. Amongst the quantum observables appearing in this paper are the lowering, raising, number and Weyl (group) operators, denoted $A, A^{+}, N$ and $W[z]$, respectively. Note that we are using the complex form for the Weyl group; the connection to the real form is $W(a, b)=$ $W[z]$ with $z=(b-i a) / \sqrt{2}$. This convention for the complex form is carried over to all functions and distributions on $\mathbb{C} \cong \mathbb{R}^{2}$. Specifically, for functions in $\mathcal{S}\left(\mathbb{R}^{2}\right), F(a, b)=F(z)=F((b-i a) / \sqrt{2})$. This is consistent with our non-standard choice of coordinates in phase space, $\Pi \cong \mathbb{R}^{2}$. A point in $\Pi$ has coordinates $(p, q)$ corresponding to momentum and position, respectively. Under the homeomorphism $\Pi \cong \mathbb{C},(p, q)$ corresponds to $(p-i q) / \sqrt{2}[4]$. 


\section{§3. Quantization}

By quantization we mean the association of an element of $\mathcal{L}\left(\mathcal{S}(\mathbb{R}), \mathcal{S}^{\prime}(\mathbb{R})\right)$ with a tempered distribution $T \in \mathcal{S}^{\prime}(\Pi)$ on phase space, $\Pi \cong \mathbb{R}^{2}$. In particular, we use the association due originally to Weyl, which we refer to simply as quantization. Our conventions are as in [4] and our formalism is based on the Wigner transform, by which we mean the mapping $\mathcal{G}: \mathcal{S}\left(\mathbb{R}^{2}\right) \rightarrow \mathcal{S}(\Pi)$ given by the formula

$$
\mathcal{G}(F)(p, q)=\frac{1}{2 \pi} \int_{\mathbb{R}} F\left(q+\frac{1}{2} u, q-\frac{1}{2} u\right) e^{i p u} d u, \quad F \in \mathcal{S}\left(\mathbb{R}^{2}\right) .
$$

(This nomenclature is not entirely standard.) It is bicontinuous and invertible, its inverse being given by $\mathcal{G}^{-1}: \mathcal{S}(\Pi) \rightarrow \mathcal{S}\left(\mathbb{R}^{2}\right)$,

$$
\mathcal{G}^{-1}(H)(x, y)=\int_{\mathbb{R}} H\left(v, \frac{1}{2}(x+y)\right) e^{-i v(x-y)} d v, \quad H \in \mathcal{S}(\Pi) .
$$

To every distribution $T \in \mathcal{S}^{\prime}(\Pi)$ corresponds a mapping $\boldsymbol{\Delta}[T] \in \mathcal{L}(\mathcal{S}(\mathbb{R})$, $\left.\mathcal{S}^{\prime}(\mathbb{R})\right)$, defined by the fundamental formula

$$
\llbracket \boldsymbol{\Delta}[T] g, \bar{f} \rrbracket=\llbracket T, \mathcal{G}(\bar{f} \otimes g) \rrbracket .
$$

The mapping $\boldsymbol{\Delta}: \mathcal{S}^{\prime}(\Pi) \rightarrow \mathcal{L}\left(\mathcal{S}(\mathbb{R}), \mathcal{S}^{\prime}(\mathbb{R})\right)$ is a bicontinuous linear bijection. Hence it is surjective, and so, given $X \in \mathcal{L}\left(\mathcal{S}(\mathbb{R}), \mathcal{S}^{\prime}(\mathbb{R})\right)$, there is a unique $T \in \mathcal{S}^{\prime}(\Pi)$ such that $X=\boldsymbol{\Delta}[T]$, and conversely. Our terminology is that $\boldsymbol{\Delta}$ is the quantization map, $\boldsymbol{\Delta}[T]$ is the quantization of $T$, and $T$ is the symbol of $\boldsymbol{\Delta}[T]$. Hence every $X \in \mathcal{L}\left(\mathcal{S}(\mathbb{R}), \mathcal{S}^{\prime}(\mathbb{R})\right)$ has a unique symbol. See [4] for details.

In fact, Weyl worked, not with $T$, but its Fourier transform, and effectively arrived at the symbolic formula (without specifying the class of functions to which it applied)

$$
\boldsymbol{\Delta}[T]=\frac{1}{2 \pi} \int_{\mathbb{C}} \mathcal{F}(T)(z) W[z] d A(z) .
$$

Our convention for the Fourier transform is as follows. Letting $n=1$ or 2 (the only cases that will occur), $\mathcal{F}: \mathcal{S}\left(\mathbb{R}^{n}\right) \rightarrow \mathcal{S}\left(\mathbb{R}^{n}\right)$ denotes the continuous automorphism

$$
[\mathcal{F} f](k)=(2 \pi)^{-n / 2} \int_{\mathbb{R}^{n}} f(x) e^{-i x \cdot k} d^{n} x, \quad f \in \mathcal{S}\left(\mathbb{R}^{n}\right) .
$$

The inverse mapping $\mathcal{F}^{-1}: \mathcal{S}\left(\mathbb{R}^{n}\right) \rightarrow \mathcal{S}\left(\mathbb{R}^{n}\right)$ is obtained, as usual, by replacing $e^{-i x \cdot k}$ with $e^{i x \cdot k}$. These maps extend continuously to unitary automorphisms 
of $L^{2}\left(\mathbb{R}^{n}\right)$, and, by a further continuous extension, to $\mathcal{S}^{\prime}\left(\mathbb{R}^{n}\right)$. In this, due care must be taken of the anti-linear embedding of $L^{2}\left(\mathbb{R}^{n}\right)$ into $\mathcal{S}^{\prime}\left(\mathbb{R}^{n}\right)$. We use the notation $\mathcal{F}, \mathcal{F}^{-1}$ in all cases.

As a central role is played by the matrix elements $\langle f, W[z] g\rangle$ of the Weyl group in what follows, we introduce a symbol for it.

Definition 3.1. By $x_{f, g}$ we mean the function

$$
x_{f, g}(z)=\langle f, W[z] g\rangle
$$

for all $f, g \in \mathcal{S}(\mathbb{R})$.

The action of the ladder operators on the test functions may be replaced with first order differential operators in the variable $z$ :

Lemma 3.1. For all $f, g \in \mathcal{S}(\mathbb{R})$ and $z \in \mathbb{C}$,

$$
\begin{aligned}
& \partial X_{f, g}=\frac{i}{2} x_{f, A g}+\frac{i}{2} X_{A^{+} f, g}, \\
& \bar{\partial} X_{f, g}=\frac{i}{2} x_{f, A^{+} g}+\frac{i}{2} X_{A f, g}, \\
& z X_{f, g}=-i X_{f, A^{+} g}+i X_{A f, g}, \\
& \bar{z} X_{f, g}=i X_{f, A g}-i X_{A^{+}, g} .
\end{aligned}
$$

The proof consists of a straightforward calculation and we omit it.

Here we have used the Wirtinger calculus, where $z$ and $\bar{z}$ are treated as independent variables, with $\bar{\partial}=\partial / \partial \bar{z}$.

Proposition 3.1. For all $f, g \in \mathcal{S}(\mathbb{R}), \chi_{f, g} \in \mathcal{S}\left(\mathbb{R}^{2}\right)$. Hence we may view $X_{f, g}$ as the value of a continuous mapping $X: \mathcal{S}(\mathbb{R}) \widehat{\otimes} \mathcal{S}(\mathbb{R}) \rightarrow \mathcal{S}\left(\mathbb{R}^{2}\right)$ at $\bar{f} \otimes g:$

$$
\chi(\bar{f} \otimes g)=X_{f, g} .
$$

Proof. Referring to the seminorms $\left\{Z_{j k m n}: j, k, m, n \in \mathbb{Z}^{+}\right\}$given in (A.4), with the help of the previous lemma we obtain

$$
Z_{j k m n}\left(X_{f, g}\right) \leq 2^{j+k} \widehat{q}_{j+k+m+n}(f) \widehat{q}_{j+k+m+n}(g) .
$$

This estimate extends in standard fashion from $\bar{f} \otimes g$ to all $F \in \mathcal{S}\left(\mathbb{R}^{2}\right)$, yielding

$$
Z_{j k m n}(X(F)) \leq 2^{j+k}\left[\widehat{q}_{j+k+m+n} \widehat{\otimes} \widehat{q}_{j+k+m+n}\right](F),
$$

which legitimizes the definition of $X$. 
The form of equation (3.3) that we need here is:

Proposition 3.2. For all $f, g \in \mathcal{S}(\mathbb{R})$ and $T \in \mathcal{S}^{\prime}(\Pi)$

$$
\llbracket \boldsymbol{\Delta}[T], \bar{f} \otimes g \rrbracket=\llbracket T, \mathcal{G}(\bar{f} \otimes g) \rrbracket=\llbracket \mathcal{F} T, X_{f, g} \rrbracket .
$$

Proof. First, for any $T \in \mathcal{S}^{\prime}(\Pi)$,

$$
F \mapsto(2 \pi)^{-1} \llbracket \mathcal{F} T, X(F) \rrbracket
$$

is a continuous linear functional on $\mathcal{S}(\mathbb{R}) \widehat{\otimes} \mathcal{S}(\mathbb{R})$ (depending on $T$ ).

Second: using the identification of $\mathcal{S}(\mathbb{R}) \widehat{\otimes} \mathcal{S}(\mathbb{R})$ with the dual of $\mathcal{L}(\mathcal{S}(\mathbb{R})$, $\left.\mathcal{S}^{\prime}(\mathbb{R})\right)$ and its reflexivity, this functional defines a map $\boldsymbol{\Delta}[T]$ in $\mathcal{L}(\mathcal{S}(\mathbb{R})$, $\left.\mathcal{S}^{\prime}(\mathbb{R})\right)$ through the formula

$$
\llbracket F, \boldsymbol{\Delta}[T] \rrbracket=\llbracket \boldsymbol{\Delta}[T], F \rrbracket=(2 \pi)^{-1} \llbracket \mathcal{F} T, X(F) \rrbracket .
$$

The order of entries in the first two pairings depends on whether $F$ is viewed as a test function or a linear functional on the space of distributions; the equality is a direct result of reflexivity.

A further consequence of reflexivity is that, setting $F=\bar{f} \otimes g$ and using $(2.1)$,

$$
\llbracket \bar{f} \otimes g, \boldsymbol{\Delta}[T] \rrbracket=\llbracket \boldsymbol{\Delta}[T] g, \bar{f} \rrbracket .
$$

Then with (3.9),

$$
\llbracket \boldsymbol{\Delta}[T] g, \bar{f} \rrbracket=(2 \pi)^{-1} \llbracket \mathcal{F} T, X_{f, g} \rrbracket,
$$

and equation (3.8) now follows.

We note that if $T$ is sufficiently regular, say $\mathcal{F} T \in L^{1}\left(\mathbb{R}^{2}\right)$, then it is legitimate to write

$$
\llbracket \mathcal{F} T, X_{f, g} \rrbracket=\left\langle f,\left\{\int[\mathcal{F} T](a, b) W(a, b) d a d b\right\} g\right\rangle .
$$

The following little result is rather useful, and we include a proof for the sake of completeness.

Lemma 3.2. The linear span of $W[\mathbb{C}]$ is dense in $\mathcal{L}\left(\mathcal{S}(\mathbb{R}), \mathcal{S}^{\prime}(\mathbb{R})\right)$.

Proof. Suppose that $F \in \mathcal{S}(\mathbb{R}) \widehat{\otimes} \mathcal{S}(\mathbb{R})$ is such that, for all $z \in \mathbb{C}$, $\llbracket F, W[z] \rrbracket=0$. We show that $F=0$ in consequence. Since

$$
\llbracket \mathcal{X}(f \otimes g) \rrbracket(z)=X_{\bar{f}, g}(z)=\llbracket f \otimes g, W[z] \rrbracket,
$$


it follows from (3.9) and the continuity of $X$ that

$$
\llbracket \boldsymbol{\Delta}[T], F \rrbracket=(2 \pi)^{-1} \llbracket \mathcal{F} T, X(F) \rrbracket=0
$$

for all $T \in \mathcal{S}^{\prime}(\Pi)$. Since the range of $\boldsymbol{\Delta}$ is $\mathcal{L}\left(\mathcal{S}(\mathbb{R}), \mathcal{S}^{\prime}(\mathbb{R})\right)$, for any $Y \in$ $\mathcal{L}\left(\mathcal{S}(\mathbb{R}), \mathcal{S}^{\prime}(\mathbb{R})\right)$

$$
\llbracket Y, F \rrbracket=0,
$$

and so $F=0$. Thus the set $\{W[z]: z \in \mathbb{C}\}$ has dense linear span in $\mathcal{L}(\mathcal{S}(\mathbb{R})$, $\left.\mathcal{S}^{\prime}(\mathbb{R})\right)$.

While $\mathcal{L}\left(\mathcal{S}(\mathbb{R}), \mathcal{S}^{\prime}(\mathbb{R})\right)$ is not an algebra, certain products do exist in some sense or another. In particular, we shall use a weak definition of products $Y X$ for arbitrary $X \in \mathcal{L}\left(\mathcal{S}(\mathbb{R}), \mathcal{S}^{\prime}(\mathbb{R})\right)$ and $Y$ a polynomial in $A$ and $A^{+}$. The construction is based on certain linear combinations of the operations introduced in equations (3.6a) $-(3.6 \mathrm{~d})$.

Lemma 3.3. Defining the four linear endomorphisms $Z_{1}, \ldots, Z_{4}$ of $\mathcal{S}\left(\mathbb{R}^{2}\right)$ by

$$
\begin{aligned}
& {\left[Z_{1} F\right](z)=-i\left(\partial+\frac{\bar{z}}{2}\right) F(z),} \\
& {\left[Z_{2} F\right](z)=-i\left(\bar{\partial}+\frac{z}{2}\right) F(z),} \\
& {\left[Z_{3} F\right](z)=-i\left(\bar{\partial}-\frac{z}{2}\right) F(z),} \\
& {\left[Z_{4} F\right](z)=-i\left(\partial-\frac{\bar{z}}{2}\right) F(z),}
\end{aligned}
$$

it follows that

$$
\begin{aligned}
& Z_{1} x_{f, g}=x_{f, A g}, \\
& Z_{2} x_{f, g}=x_{A f, g}, \\
& Z_{3} x_{f, g}=x_{f, A^{+} g}, \\
& Z_{4} x_{f, g}=x_{A^{+}, g}, .
\end{aligned}
$$

The proof is immediate.

To transfer these endomorphisms to act on distributions in phase space we combine duality and Fourier transform. In this regard, our notation for the dual of an element $X \in \mathcal{L}\left(\mathcal{S}(\mathbb{R}), \mathcal{S}^{\prime}(\mathbb{R})\right)$ is $X^{\mathrm{tr}}$.

Proposition 3.3. The 'conjugate' endomorphisms $\mathcal{Z}_{j}$ of $\mathcal{S}^{\prime}(\Pi)(j=1$, $2,3,4)$

$$
\mathcal{Z}_{j}=\mathcal{F}^{-1} Z_{j}^{\mathrm{tr}} \mathcal{F}
$$


are such that, for any $T \in \mathcal{S}^{\prime}(\Pi)$,

$$
\begin{aligned}
& \boldsymbol{\Delta}\left[\mathcal{Z}_{1} T\right]=\boldsymbol{\Delta}[T] A, \\
& \boldsymbol{\Delta}\left[\mathcal{Z}_{2} T\right]=A^{\mathrm{tr}} \boldsymbol{\Delta}[T], \\
& \boldsymbol{\Delta}\left[\mathcal{Z}_{3} T\right]=\boldsymbol{\Delta}[T] A^{+}, \\
& \boldsymbol{\Delta}\left[\mathcal{Z}_{4} T\right]=\left[A^{+}\right]^{\operatorname{tr}} \boldsymbol{\Delta}[T] .
\end{aligned}
$$

Again, for the sake of brevity we omit the elementary calculations.

Note that this solves the problem of defining $Y X$ outlined above, since every element of $\mathcal{L}\left(\mathcal{S}(\mathbb{R}), \mathcal{S}^{\prime}(\mathbb{R})\right)$ is of the form $\boldsymbol{\Delta}[T]$.

\section{§4. The Dilated System, Kinematics}

The oscillator in this model is an open system: it is subject to external influences. Hence its time translations are not implemented by unitary operators. One can model the external influences in different ways, and we have chosen to use the description based on a quantum Wiener process in the sense of Hudson and Parthasaraty, [8]. To avoid circumlocutions, we will refer to the oscillator as the 'system', the Wiener process as the 'reservoir' and the combination of the two as the 'universe' - accepting that these are misnomers.

The general procedure we are following is a standard one for open systems. We construct the universe on the usual basis of tensor products, and then impose a time translation scheme that entangles the system and reservoir. The generator of the time translations consists of free evolution for system and reservoir, plus an interaction term, and overall, is not Hamiltonian. The generator is of Lindblad type, as it must be, but as it is an unbounded (discontinuous) mapping, we cannot turn to known mathematical results - there are none which are relevant. We do know that when the observables are bounded operators or polynomials in $A, A^{+}$and $N$, the problem has been solved by Alli and Sewell [7], following on earlier work of Hepp and Lieb [9]. It remains, however, to extend the Alli-Sewell results to all of $\mathcal{L}\left(\mathcal{S}(\mathbb{R}), \mathcal{S}^{\prime}(\mathbb{R})\right)$.

In this section we shall construct the reservoir and universe; in the next section we begin our construction of the dynamics.

The quantum Wiener process we use is based on the symmetric Fock space over the one particle Hilbert space $\mathfrak{h}=L^{2}([0, \infty))$. As well as $\mathfrak{h}$, we need its closed unit ball, denoted $\mathfrak{b}$ : a function $f \in \mathfrak{h}$ belongs to $\mathfrak{b}$ if $\|f\| \leq 1$.

The $n$ particle Hilbert space $\mathfrak{h}_{n}$ will be the symmetrized $n$-fold Hilbertian tensor product of $\mathfrak{h}$ with itself; as usual, $\mathfrak{h}_{0}=\mathbb{C}$ is the ground field. By $\widetilde{\mathcal{H}}^{(0)}$ we mean the algebraic direct sum $\oplus_{n \geq 0} \mathfrak{h}_{n}$, the incomplete Fock space. An 
element $\left(\Phi_{n}\right)$ of this space is a terminating sequence: $\Phi_{n} \in \mathfrak{h}_{n}$ and there is an integer $K$ such that $\Phi_{k}=0$ for all $k>K$. The Hilbertian completion of $\widetilde{\mathcal{H}}^{(0)}$ is the Fock space, denoted $\widetilde{\mathcal{H}}$.

The free Bose field for this process, $\widetilde{w}$, is taken to have the incomplete Fock space $\widetilde{\mathcal{H}}^{(0)}$ as its domain. Then $\widetilde{w}(f)$ and $\widetilde{w}(f)^{+}$are endomorphisms of $\widetilde{\mathcal{H}}^{(0)}$ for any $f \in \mathfrak{h}$, and satisfy the canonical commutation relations strongly on this domain: for any $f, g \in \mathfrak{h}$ and $\Phi \in \widetilde{\mathcal{H}}^{(0)}$,

$$
\widetilde{w}(f) \widetilde{w}(g)^{+} \Phi-\widetilde{w}(g)^{+} \widetilde{w}(f) \Phi=\langle g, f\rangle \Phi .
$$

We denote the corresponding Weyl operators $\widetilde{W}(f)$, and these unitary operators act on the Fock space $\widetilde{\mathcal{H}}$ :

$$
\widetilde{W}(f)=\exp \left[i\left(\widetilde{w}(f)+\widetilde{w}(f)^{+}\right)\right] .
$$

We have referred to this scheme as a quantum Wiener process for the following reason: the 'independent variable' for this field has the character of a time, and the field itself may be used to define conditional expectations. These are based on the supports of the test functions, as discussed by Alli and Sewell [7]. However, we shall not need this probabilistic structure here.

The canonical Fock vector is $\widetilde{\Omega}=(1,0,0, \ldots)$, and is cyclic for the algebra of polynomials in the field $\widetilde{w}$. Hence $\widetilde{\Omega}$ defines a state on $\mathcal{L}(\widetilde{\mathcal{H}})$, which we take as the reference state for projecting down from the universe to the oscillator.

There are a number of different possible 'test function' space for the universe. First of all there is the space in which the oscillator test function space is complete, but the reservoir space is not:

$$
\widehat{\mathcal{Q}}^{(0)}=\mathcal{S}(\mathbb{R}) \otimes \widetilde{\mathcal{H}}^{(0)} .
$$

The technical advantage here is that the elements of $\widetilde{\mathcal{H}}^{(0)}$ are finite sequences.

We equip this space with the seminorms $\left\{q_{r s}: r, s \in \mathbb{Z}^{+}\right\}$, see (A.5a); evidently $\widehat{\mathcal{Q}}^{(0)}$ is not complete in the topology determined by these seminorms. Its completion is denoted $\widehat{\mathcal{Q}}$.

Correspondingly, the system Hilbert space for the universe is $\widehat{\mathcal{H}}=$ $L^{2}(\mathbb{R}) \widehat{\otimes}_{h} \widetilde{\mathcal{H}}$, so the distinguished rigged space for the universe is

$$
\widehat{\mathcal{Q}} \subset \widehat{\mathcal{H}} \subset \widehat{\mathcal{Q}}^{\prime} .
$$

We note that the completion of $\widehat{\mathcal{Q}}^{(0)}$ in the Hilbertian topology is $\widehat{\mathcal{H}}$.

Mappings of the form $X \otimes I$ and $I \otimes Y\left(X \in \mathcal{L}\left(\mathcal{S}(\mathbb{R}), \mathcal{S}^{\prime}(\mathbb{R})\right), Y \in \mathcal{L}(\widetilde{\mathcal{H}})\right.$ are (generalized) observables localized in the oscillator and reservoir subsystems 
respectively. In particular, the maps $A \otimes I, A^{+} \otimes I, I \otimes \widetilde{w}(f), I \otimes \widetilde{w}(f)^{+}$for $f \in \mathfrak{h}$, are continuous endomorphisms of $\widehat{\mathcal{Q}}$.

\section{$\S 5 . \quad$ Dilated Dynamics}

The reservoir is dynamically coupled to the oscillator in a rather special way, mediated with the help of the functions $h_{t} \in \mathfrak{h}(t \geq 0)$ given by

$$
h_{t}(s)=g_{0} \sqrt{2 \pi} e^{-\zeta(t-s)} \chi_{[0, t]}(s),
$$

so that

$$
\left\|h_{t}\right\|^{2}=1-\exp \left(-2 \pi g_{0}^{2} t\right)
$$

For interpretive purposes, $\zeta=\pi g_{0}^{2}+i \omega$ is the (complex) frequency of the damped oscillator. The constant $\omega$ is the frequency of the undriven oscillator and $g_{0}$ has the character of a coupling constant to the otherwise unspecified external forces.

Definition 5.1. By $G_{t}(z)$ we mean the Gaussian function

$$
G_{t}(z)=\exp \left[-\frac{1}{2}|z|^{2}\left(1-e^{-2 \pi g_{0}^{2} t}\right)\right]=\exp \left[-\frac{1}{2}|z|^{2}\left\|h_{t}\right\|^{2}\right], \quad t \geq 0, z \in \mathbb{C} .
$$

We take from Alli and Sewell [7] their result for the unitary dynamics covering mappings of the form $P \otimes I$, where $P$ is an element of the *-algebra generated by the bounded operators and polynomials in $A$ and $A^{+}$. By restriction, this gives us a weakly continuous one parameter semigroup $\left\{\widehat{T}_{t}^{(A S)}: t \geq 0\right\}$ of contractive ${ }^{*}$-isomorphisms of $\mathcal{L}(\widehat{\mathcal{H}})$, whose action on $W[z]$ is given by

$$
\widehat{T}_{t}^{(A S)}(W[z] \otimes I)=W\left[e^{-\zeta t} z\right] \otimes \widetilde{W}\left(z h_{t}\right),
$$

entangling oscillator and reservoir.

This formula will be recognized as equivalent to the solution of the quantum Langevin equation (dropping the tensor product signs for clarity)

$$
\widehat{T}_{t}^{(A S)}(A)-A+i \zeta \int_{0}^{t} \widehat{T}_{u}^{(A S)}(A) d u=\sqrt{2 \pi} g_{0} \widetilde{w}\left(\chi_{[0, t]}\right)
$$

Here $\chi_{[0, t]}$ is the characteristic function of the real set $[0, t]$.

Remark. We are taking the formulas for $U_{t}$ acting on the ladder operators from Alli and Sewell [7]. All questions of continuity in this regard will be addressed $a b$ initio as part of the analysis below, Propositions 5.1, 5.2. 
We are going to use $h_{t}$ to construct a dense subspace $\mathfrak{m}$ of $\mathfrak{h}$, introduced as a technical tool so that certain calculations can be effected.

Definition 5.2. $\quad$ By $\mathfrak{m}$ we mean the dense linear subspace of $\mathfrak{h}$ which is the finite linear span of the set $\left\{\sigma_{s} h_{t}: s, t \geq 0\right\}$, where, for any $t \geq 0$, the map $\sigma_{t} \in \mathcal{L}(\mathfrak{h})$ is given by

$$
\left[\sigma_{t} f\right](s)= \begin{cases}0, & 0 \leq s \leq t \\ f(s-t), & s \geq t\end{cases}
$$

Observe that, if $f \in \mathfrak{m}^{\perp}$, then for all $t \geq 0$,

$$
0=\left\langle f, h_{t}\right\rangle=\sqrt{2 \pi} g_{0} e^{-\zeta t} \int_{0}^{t} \overline{f(s)} e^{\zeta s} d s
$$

hence $f=0$.

Remark. While $\lim _{t \rightarrow 0} \sigma_{t}=I$ strongly in $\mathcal{L}(\mathfrak{h})$, this convergence is not valid in the operator norm topology, a fact which has significant implications later on.

The following proposition enables us to replace functions in $\mathfrak{h}$ with the more tractable functions in $\mathfrak{m}$ in the seminorms $p_{\text {rs }}$ of equation (A.5b), and still determine the same locally convex topology on $\hat{\mathcal{Q}}^{(0)}$ and its completion $\hat{\mathcal{Q}}$. For if we define the seminorms

$$
p_{r s}^{(0)}(\widehat{\Phi})=\sup \left\{\left\|A^{r} \otimes \widetilde{w}\left(f_{1}\right) \cdots \widetilde{w}\left(f_{s}\right) \widehat{\Phi}\right\|: f_{1}, \ldots, f_{s} \in \mathfrak{b} \cap \mathfrak{m}\right\},
$$

using $\mathfrak{m}$, then

Lemma 5.1. For all $\widehat{\Phi} \in \widehat{\mathcal{Q}}, r, s \in \mathbb{Z}^{+}$,

$$
p_{r s}^{(0)}(\widehat{\Phi})=p_{r s}(\widehat{\Phi}) \text {. }
$$

Proof. It is clear that $p_{r s}^{(0)}(\widehat{\Phi}) \leq p_{r s}(\widehat{\Phi})$, so we must prove the opposite inequality.

Given a family $\left\{f_{1}, \ldots, f_{s}\right\}$ in $\mathfrak{b}$, for each $j(1 \leq j \leq s)$ we can find a sequence $\left(g_{j}^{(n)}\right)$ in $\mathfrak{m} \cap \mathfrak{b}$ such that $g_{j}^{(n)} \rightarrow f_{j}$ as $n \rightarrow \infty$ in the norm on $\mathfrak{h}$. As the $g_{j}^{(n)} \in \mathfrak{b}$, they are accounted in the supremum for $p_{r s}^{(0)}$, and so, for all $n \in \mathbb{N}$,

$$
\left\|A^{r} \otimes \widetilde{w}\left(g_{1}^{(n)}\right) \cdots \widetilde{w}\left(g_{s}^{(n)}\right) \widehat{\Phi}\right\| \leq p_{r s}^{(0)}(\widehat{\Phi}) .
$$


To pass to the limit $n \rightarrow \infty$, we compare the two constructions: for all $n \in \mathbb{N}$,

$$
\begin{aligned}
& \left\|A^{r} \otimes \widetilde{w}\left(g_{1}^{(n)}\right) \cdots \widetilde{w}\left(g_{s}^{(n)}\right) \widehat{\Phi}-A^{r} \otimes \widetilde{w}\left(f_{1}\right) \cdots \widetilde{w}\left(f_{s}\right) \widehat{\Phi}\right\| \\
& \leq \sum_{j=1}^{s}\left\|A^{r} \otimes \widetilde{w}\left(f_{1}^{(n)}\right) \cdots \widetilde{w}\left(f_{j-1}\right) \widetilde{w}\left(f_{j}-g_{j}^{(n)}\right) \widetilde{w}\left(g_{j+1}^{(n)}\right) \cdots \widetilde{w}\left(g_{s}^{(n)}\right) \widehat{\Phi}\right\| \\
& \quad \leq\left(\sum_{j=1}^{s}\left\|f_{j}-g_{j}^{(n)}\right\|\right) p_{r s}(\widehat{\Phi}) .
\end{aligned}
$$

Therefore

$$
\lim _{n \rightarrow \infty}\left\|A^{r} \otimes \widetilde{w}\left(g_{1}^{(n)}\right) \cdots \widetilde{w}\left(g_{s}^{(n)}\right) \widehat{\Phi}\right\|=\left\|A^{r} \otimes \widetilde{w}\left(f_{1}\right) \cdots \widetilde{w}\left(f_{s}\right) \widehat{\Phi}\right\| .
$$

Each term in the sequence has been bounded above by $p_{r, s}^{(0)}(\widehat{\Phi})$, and it follows, therefore, that

$$
\left\|A^{r} \otimes \widetilde{w}\left(f_{1}\right) \cdots \widetilde{w}\left(f_{s}\right) \widehat{\Phi}\right\| \leq p_{r, s}^{(0)}(\widehat{\Phi}),
$$

completing the proof.

Proposition 5.1 [Alli and Sewell]. The mappings $T_{t}^{(A S)}$ are unitarily implemented: for all $X \in \mathcal{L}(\widehat{\mathcal{H}})$, there exists a unitary map $U_{t}$ on $\widehat{\mathcal{H}}$ such that

$$
T_{t}^{(A S)}(X)=U_{t}^{*} X U_{t}
$$

Moreover, for all $f \in \mathfrak{m}, \widehat{\Phi} \in \widehat{\mathcal{Q}}$ and all $t \geq 0$,

$$
\begin{aligned}
U_{t}^{*}(A \otimes I) U_{t} \widehat{\Phi} & =e^{-\zeta t}(A \otimes I) \widehat{\Phi}+\left(I \otimes \widetilde{w}\left(h_{t}\right)\right) \widehat{\Phi}, \\
U_{t}^{*}(I \otimes \widetilde{w}(f)) U_{t} \widehat{\Phi} & =\left(I \otimes \widetilde{w}\left(\sigma_{t} f\right)\right) \widehat{\Phi} .
\end{aligned}
$$

Proof. Alli and Sewell [7] proved equation (5.9a) for $f=h_{s}$, and our result follows by finite linear combination.

Proposition 5.2. The map $U_{t} \in \mathcal{L}(\widehat{\mathcal{Q}})$, with the uniform (in $\left.t\right)$ bound

$$
p_{r, s}\left(U_{t} \widehat{\Phi}\right) \leq 2^{r} \hat{p}_{r, s+r}(\widehat{\Phi}), \quad \widehat{\Phi} \in \widehat{\mathcal{Q}}, r, s \in \mathbb{Z}^{+} .
$$

Proof. For all $f_{1}, \ldots, f_{s} \in \mathfrak{m}, \widehat{\Phi} \in \widehat{\mathcal{Q}}$ and $t \geq 0$,

$$
\begin{aligned}
\left(A^{r}\right. & \left.\otimes \widetilde{w}\left(f_{1}\right) \otimes \cdots \otimes \widetilde{w}\left(f_{s}\right)\right) U_{t} \widehat{\Phi} \\
& =\sum_{j=0}^{r}\left(\begin{array}{l}
r \\
j
\end{array}\right) e^{-j \zeta t} U_{t}\left(A^{j} \otimes \widetilde{w}\left(h_{t}\right)^{r-j} \widetilde{w}\left(\sigma_{t} f_{1}\right) \cdots \widetilde{w}\left(\sigma_{t} f_{s}\right)\right) \widehat{\Phi} .
\end{aligned}
$$


Then

$$
\begin{aligned}
p_{r s}\left(U_{t} \widehat{\Phi}\right) & =p_{r s}^{(0)}\left(U_{t} \widehat{\Phi}\right) \\
& \leq \sum_{j=0}^{r}\left(\begin{array}{l}
r \\
j
\end{array}\right) p_{j, s+r-j}^{(0)}(\widehat{\Phi})=\sum_{j=0}^{r}\left(\begin{array}{l}
r \\
j
\end{array}\right) p_{j, s+r-j}(\widehat{\Phi}) \\
& \leq 2^{r} \widehat{p}_{r, s+r}(\widehat{\Phi}) .
\end{aligned}
$$

The assertion of the proposition is now immediate.

We extend the domain of $U_{t}$ to include fields $\widetilde{w}(f)$ with $f \in \mathfrak{h}$ in the following sense:

Proposition 5.3. For any $\widehat{\Phi}, \widehat{\Psi} \in \widehat{\mathcal{Q}}, f \in \mathfrak{h}$ and $t \geq 0$,

$$
\left\langle U_{t} \widehat{\Phi},(I \otimes \widetilde{w}(f)) U_{t} \widehat{\Psi}\right\rangle=\left\langle\widehat{\Phi},\left(I \otimes \widetilde{w}\left(\sigma_{t} f\right)\right) \widehat{\Psi}\right\rangle .
$$

Proof. For any $\widehat{\Phi}, \widehat{\Psi} \in \widehat{\mathcal{Q}}, f \in \mathfrak{h}$ and $t \geq 0$, the two inequalities

$$
\begin{aligned}
\left|\left\langle U_{t} \widehat{\Phi},(I \otimes \widetilde{w}(f)) U_{t} \widehat{\Psi}\right\rangle\right| & \leq\|\widehat{\Phi}\| p_{01}\left(U_{t} \widehat{\Psi}\right)\|f\| \leq\|\widehat{\Phi}\| \widehat{p}_{01}(\widehat{\Psi})\|f\| \\
\left|\left\langle\widehat{\Phi},\left(I \otimes \widetilde{w}\left(\sigma_{t} f\right)\right) \widehat{\Psi}\right\rangle\right| & \leq\|\widehat{\Phi}\| p_{01}(\widehat{\Psi})\left\|\sigma_{t} f\right\| \leq\|\widehat{\Phi}\| p_{01}(\widehat{\Psi})\|f\|
\end{aligned}
$$

hold. Furthermore, for any $\widehat{\Phi}, \widehat{\Psi} \in \widehat{\mathcal{Q}}$ and $t \geq 0$, the two maps

$$
\begin{aligned}
& f \mapsto\left\langle U_{t} \widehat{\Phi},(I \otimes \widetilde{w}(f)) U_{t} \widehat{\Psi}\right\rangle, \\
& f \mapsto\left\langle\widehat{\Phi},\left(I \otimes \widetilde{w}\left(\sigma_{t} f\right)\right) \widehat{\Psi}\right\rangle,
\end{aligned}
$$

are continuous linear functionals on $\mathfrak{h}$ which agree on $\mathfrak{m}$. Hence they must be equal, completing the proof.

Corollary 5.1. For any $X \in \mathcal{L}\left(\widehat{\mathcal{Q}},(\widehat{\mathcal{Q}})^{\prime}\right)$ and $t \geq 0$, we may define $\widehat{T}_{t}(X) \in \mathcal{L}\left(\widehat{\mathcal{Q}},(\widehat{\mathcal{Q}})^{\prime}\right)$ by setting

$$
\llbracket \widehat{T}_{t}(X) \widehat{\Psi}, \bar{\Phi} \rrbracket=\llbracket X U_{t} \widehat{\Psi}, \overline{U_{t} \widehat{\Phi}} \rrbracket, \quad \widehat{\Phi}, \widehat{\Psi} \in \widehat{\mathcal{Q}} .
$$

Moreover, $\widehat{T}_{t}$ coincides with $T_{t}^{(A S)}$ for $X=A \otimes I$ and $I \otimes \widetilde{w}(f)$ for all $f \in \mathfrak{h}$, and on $\widehat{\mathcal{Q}}$ for all $X \in \mathcal{L}(\widehat{\mathcal{H}})$.

Proof. Equation (5.12) is immediate from the previous proposition. From this equation follows the statements about $A \otimes I$ and $I \otimes \widetilde{w}(f)$. We now prove that $\widehat{T}_{t}(X)$ coincides with $T_{t}^{(A S)}(X)$ on $\widehat{\mathcal{Q}}$ for all $X \in \mathcal{L}(\widehat{\mathcal{H}})$. 
If $X \in \mathcal{L}(\widehat{\mathcal{H}})$, we identify it with an element in $\mathcal{L}\left(\widehat{\mathcal{Q}},(\widehat{\mathcal{Q}})^{\prime}\right)$ (also written $X)$, so that, for all $\widehat{\Phi}, \widehat{\Psi} \in \widehat{\mathcal{Q}}$ and $t \geq 0$,

$$
\begin{aligned}
\llbracket \widehat{T}_{t}(X) \widehat{\Psi}, \overline{\widehat{\Phi}} \rrbracket & =\llbracket X U_{t} \widehat{\Psi}, \overline{U_{t} \widehat{\Phi} \rrbracket}=\left\langle U_{t} \widehat{\Phi}, X U_{t} \widehat{\Psi}\right\rangle \\
& =\left\langle\widehat{\Phi}, U_{t}^{*} X U_{t} \widehat{\Psi}\right\rangle=\left\langle\widehat{\Phi}, T_{t}^{(A S)}(X) \widehat{\Psi}\right\rangle .
\end{aligned}
$$

In particular, taking $X=W[z]$ and $t \geq 0$,

$$
\widehat{T}_{t}(W[z] \otimes I)=W\left[e^{-\zeta t} z\right] \otimes \widetilde{W}\left(z h_{t}\right) .
$$

The proof is now complete.

We are going to prove that $\left\{\widehat{T}_{t}: t \geq 0\right\}$ determines the time translations on $\mathcal{L}\left(\widehat{\mathcal{Q}},(\widehat{\mathcal{Q}})^{\prime}\right)$, and so constitutes the dynamics for the universe. We begin by proving that this collection of 1-parameter mappings has the semigroup property.

Proposition 5.4. The collection $\left\{\widehat{T}_{t}: t \geq 0\right\}$ is a one parameter equicontinuous family of endomorphisms of $\mathcal{L}\left(\widehat{\mathcal{Q}},(\widehat{\mathcal{Q}})^{\prime}\right)$ which satisfies the semigroup law.

Proof. The semigroup property for the $\widehat{T}_{t}$ follows from the fact that $\left\{T_{t}^{(A S)}: t \geq 0\right\}$ is a semigroup acting on $\mathcal{L}(\widehat{\mathcal{H}})$. For it is then the case that the collection $\left\{U_{t}: t \geq 0\right\}$, implementing $\left\{T_{t}^{(A S)}: t \geq 0\right\}$, has the additive property: for all $s, t \geq 0, U_{s+t} U_{t}^{*} U_{s}^{*}$ is in the centre of $\mathcal{L}(\widehat{\mathcal{H}})$. Consequently there is a complex number of modulus unity, $\lambda(s, t)$ such that

$$
U_{s+t}=\lambda(s, t) U_{t}^{*} U_{s}^{*} .
$$

Following the pattern of equation (5.13): for all $\widehat{\Phi}, \widehat{\Psi} \in \widehat{\mathcal{Q}}, X \in \mathcal{L}\left(\widehat{\mathcal{Q}},(\widehat{\mathcal{Q}})^{\prime}\right)$ and $s, t \geq 0$,

$$
\begin{aligned}
\llbracket \widehat{T}_{t+s}(X) \widehat{\Psi}, \overline{\widehat{\Phi}} \rrbracket & =\llbracket X U_{t+s} \widehat{\Psi}, \overline{U_{t+s} \widehat{\Phi}} \rrbracket=\llbracket X U_{s} U_{t} \widehat{\Phi}, \overline{X U_{s} U_{t}} \widehat{\Psi} \rrbracket \\
& =\llbracket \widehat{T}_{s}(X) U_{t} \widehat{\Psi}, \overline{U_{t} \widehat{\Phi}} \rrbracket=\llbracket \widehat{T}_{t}\left(\widehat{T}_{s}(X)\right) \widehat{\Psi}, \widehat{\widehat{\Phi}} \rrbracket
\end{aligned}
$$

Thus, for all $s, t \geq 0$,

$$
\widehat{T}_{s+t}=\widehat{T}_{s} \widehat{T}_{t}
$$

The equicontinuity follows from equation (5.10). 
Remark. We are not claiming that $\left\{\widehat{T}_{t}: t \geq 0\right\}$ is an equicontinuous one parameter semigroup of class $C_{0}$ on $\mathcal{L}\left(\widehat{\mathcal{Q}},(\widehat{\mathcal{Q}})^{\prime}\right)$. For, as noted previously, $\sigma_{t}$ does not converge to $I$ in norm as $t \rightarrow 0$. This means that we cannot show that $U_{t}$ converges to $I$ strongly in $\mathcal{L}(\widehat{\mathcal{Q}})$ as $t \rightarrow 0$. Hence we are prevented from establishing the continuity of $\widehat{T}_{t}$ as a function of $t$, which is necessary for a semigroup on a locally convex space. Nonetheless, we will be able to prove that the compressed family obtained from $\left\{\widehat{T}_{t}: t \geq 0\right\}$ has all the requisite properties, and is even differentiable. These results are obtained by our pullback to phase space.

\section{§6. Compressive Dynamics; Phase Space}

Turning now to the dynamics for the oscillator subsystem, this is to be obtained by compression. Our first concern, therefore, is the compressive projection. As a technical tool we must restrict the incomplete space $\widehat{\mathcal{Q}}^{(0)}$ by restricting the oscillator test functions to the subspace $\mathcal{S}(\mathbb{R})^{(0)}$ of $\mathcal{S}(\mathbb{R})$, consisting of finite linear combinations of Hermite-Gauss functions $\left\{h_{n}: n \in \mathbb{Z}^{+}\right\}$. Then we define

$$
\widehat{\mathcal{Q}}^{(0,0)}=\mathcal{S}(\mathbb{R})^{(0)} \otimes \widetilde{\mathcal{H}}^{(0)} .
$$

(We apologize for the surfeit of zeroes.) Evidently $\widehat{\mathcal{Q}}^{(0,0)}$ is a dense linear subspace of $\widehat{\mathcal{Q}}$.

Proposition 6.1. $\quad$ Consider the sesquilinear map $\Pi: \widehat{\mathcal{Q}}^{(0,0)} \times \widehat{\mathcal{Q}}^{(0,0)} \rightarrow$ $\mathcal{S}(\mathbb{R}) \widehat{\otimes} \mathcal{S}(\mathbb{R})$ given by linear extension from

$$
\Pi(f \otimes \widetilde{\Theta}, g \otimes \widetilde{\Gamma})=\langle\widetilde{\Theta}, \widetilde{\Gamma}\rangle \bar{f} \otimes g, \quad f, g \in \mathcal{S}(\mathbb{R})^{(0)}, \widetilde{\Theta}, \widetilde{\Gamma} \in \widetilde{\mathcal{H}}^{(0)}
$$

Then $\Pi$ extends to a map $\Pi: \widehat{\mathcal{Q}} \times \widehat{\mathcal{Q}} \rightarrow \mathcal{S}(\mathbb{R}) \widehat{\otimes} \mathcal{S}(\mathbb{R})$ such that, for all $\widehat{\Phi}, \widehat{\Psi} \in \widehat{\mathcal{Q}}$ and all $r, s \geq 0$,

$$
\left(\widetilde{p}_{r} \otimes \widetilde{p}_{s}\right)[\Pi(\widehat{\Phi}, \widehat{\Psi})] \leq \frac{\pi^{2}}{6} \widetilde{p}_{r+2,0}(\widehat{\Phi}) \widetilde{p}_{s+2,0}(\widehat{\Psi}) .
$$

No confusion is likely from our keeping the same symbol $\Pi$, for the extension map.

Proof. We need a continuity estimate enabling us to extend $\Pi$. Let $\widehat{\Phi}$, $\widehat{\Psi} \in \widehat{\mathcal{Q}}^{(0,0)}$. Then there exist $\widetilde{\Theta}_{1}, \ldots, \widetilde{\Theta}_{M}, \widetilde{\Gamma}_{1}, \ldots, \widetilde{\Gamma}_{N} \in \widetilde{\mathcal{H}}^{(0)}$ such that

$$
\widehat{\Phi}=\sum_{m=0}^{M} h_{m} \otimes \widetilde{\Theta}_{m}, \quad \widehat{\Psi}=\sum_{n=0}^{N} h_{n} \otimes \widetilde{\Gamma}_{n} .
$$


Then

$$
\begin{aligned}
\left(\widetilde{p}_{r}\right. & \left.\otimes \widetilde{p}_{s}\right)[\Pi(\widehat{\Phi}, \widehat{\Psi})] \leq \sum_{m=0}^{M} \sum_{n=0}^{N}\left|\left\langle\widetilde{\Theta}_{m}, \widetilde{\Gamma}_{n}\right\rangle\right|(m+1)^{r / 2}(n+1)^{s / 2} \\
& \leq \sum_{m=0}^{M} \sum_{n=0}^{N}\left\|\widetilde{\Theta}_{m}\right\|\left\|\widetilde{\Gamma}_{n}\right\|(m+1)^{r / 2}(n+1)^{s / 2} \\
& \leq \frac{\pi^{2}}{6}\left(\sum_{m=0}^{M}(m+1)^{r+2}\|\widetilde{\Theta}\|^{2}\right)^{1 / 2}\left(\sum_{n=0}^{N}(n+1)^{s+2}\|\widetilde{\Gamma}\|^{2}\right)^{1 / 2} \\
& =\frac{\pi^{2}}{6} \widetilde{p}_{r+2,0}(\widehat{\Phi}) \widetilde{p}_{s+2,0}(\widehat{\Psi}) .
\end{aligned}
$$

Equation (6.3) is now immediate, and with it the completion of the proof.

We are going to compress $\widehat{T}_{t}$, acting on observables in the universe, to $T_{t}$, acting on observables in the oscillator subsystem, in the usual way for open systems.

Definition 6.1. For $t \in[0, \infty)$, let $T_{t}$ be the endomorphism of $\mathcal{L}(\mathcal{S}(\mathbb{R})$, $\left.\mathcal{S}^{\prime}(\mathbb{R})\right)$ given as follows: for all $X \in \mathcal{L}\left(\mathcal{S}(\mathbb{R}), \mathcal{S}^{\prime}(\mathbb{R})\right)$ and $f, g \in \mathcal{S}(\mathbb{R}), t \geq 0$,

$$
\llbracket T_{t}(X) g, f \rrbracket=\llbracket \widehat{T}_{t}(X \otimes I)(g \otimes \widetilde{\Omega}), f \otimes \widetilde{\Omega} \rrbracket .
$$

We must now prove that $\left\{T_{t}: t \geq 0\right\}$ is the dynamical semigroup for the (damped) oscillator, effecting an open dynamics. As mentioned above, the vector state corresponding to $\widetilde{\Omega}$ acts as a reference state in this procedure.

The first result for the $\left\{T_{t}: t \geq 0\right\}$ that we obtain is that this collection of one parameter mappings is, in fact, a locally equicontinuous semigroup of class $C_{0}$.

As mentioned several times, we are able to do this by transferring the dynamical law to tempered distributions on phase space. More specifically, we will consider the collection $\left\{C_{t}^{\dagger}: t \geq 0\right\}$ of endomorphisms of $\mathcal{S}^{\prime}(\Pi)$ defined in such a way that

$$
T_{t}(\boldsymbol{\Delta}[T])=\boldsymbol{\Delta}\left[C_{t}^{\dagger} T\right] .
$$

In a usual way, endomorphisms of tempered distributions are defined by duality. Hence we must consider the 'pre-dual' mappings corresponding to the $C_{t}^{\dagger}$. More precisely, we consider the endomorphisms of test functions conjugated by the Fourier transform, which is the reason for the dagger notation. This enables us to use the Wirtinger calculus results we previously obtained for the $Z_{j}$ and the $z_{j}$, equations $(3.10 \mathrm{a})-(3.10 \mathrm{~d})$ and $(3.13 \mathrm{a})-(3.13 \mathrm{~d})$ respectively. 
Proposition 6.2. The set $\left\{C_{t}: t \geq 0\right\}$ of endomorphisms of $\mathcal{S}\left(\mathbb{R}^{2}\right)$ given by (see equation (5.2) for $G_{t}$ )

$$
\left[C_{t} F\right](z)=G_{t}(z) F\left(e^{-\zeta t} z\right), \quad F \in \mathcal{S}\left(\mathbb{R}^{2}\right),
$$

is a locally equicontinuous one parameter semigroup of class $C_{0}$ acting on $\mathcal{S}\left(\mathbb{R}^{2}\right)$.

Proof. For any $t \geq 0$, evidently $C_{t} \in \mathcal{L}\left(\mathcal{S}\left(\mathbb{R}^{2}\right)\right)$. The semigroup property results from the identity

$$
G_{s}(z) G_{t}\left(e^{-s \zeta} z\right)=G_{s+t}(z)
$$

holding for all $s, t \geq 0$ and $z \in \mathbb{C}$. We take it as obvious that $t \mapsto C_{t} F$ from $[0, \infty)$ to $\mathcal{S}\left(\mathbb{R}^{2}\right)$ is continuous for any $F \in \mathcal{S}\left(\mathbb{R}^{2}\right)$.

For any $F \in \mathcal{S}\left(\mathbb{R}^{2}\right), \tau>0$ and $j, k, m, n \in \mathbb{Z}^{+}$, we can find $j^{\prime}, k^{\prime}, m^{\prime}$, $n^{\prime} \in \mathbb{Z}^{+}$and a constant $K>0$ such that (see (A.4)

$$
Z_{j k m n}\left(C_{s} F-C_{t} F\right) \leq K Z_{j^{\prime} k^{\prime} m^{\prime} n^{\prime}}(F)|s-t|, \quad 0 \leq s, t \leq \tau .
$$

This is the local equi-continuity property, which completes the proof.

Turning to the conjugate dual mapping, given by

Proposition 6.3. The set $\left\{C_{t}^{\dagger}: t \geq 0\right\}$ of endomorphisms of $\mathcal{S}^{\prime}(\Pi)$

$$
C_{t}^{\dagger}=\mathcal{F}^{-1} C_{t}{ }^{\mathrm{tr}} \mathcal{F}
$$

is a locally equicontinuous one parameter semigroup of class $C_{0}$ acting on $\mathcal{S}^{\prime}(\Pi)$.

Proof. The collection $\left\{C_{t}^{\dagger}: t \geq 0\right\}$ has the semigroup property because $\left\{C_{t}: t \geq 0\right\}$ does.

From the continuity properties of the Fourier transform, it is clear that $C_{t}^{\dagger} \in \mathcal{L}\left(\mathcal{S}^{\prime}(\Pi)\right)$. For the same reason, it follows that $t \mapsto C_{t}^{\dagger} T$ from $[0, \infty)$ to $\mathcal{S}^{\prime}(\Pi)$ is continuous for any $T \in \mathcal{S}^{\prime}(\Pi)$.

For all $F \in \mathcal{S}\left(\mathbb{R}^{2}\right), T \in \mathcal{S}^{\prime}(\Pi)$ and $t \geq 0$,

$$
\llbracket \mathcal{F} C_{t}^{\dagger} T, F \rrbracket=\llbracket \mathcal{F} T, C_{t} F \rrbracket .
$$

Therefore

$$
\llbracket \mathcal{F}\left(C_{s}^{\dagger} T-C_{t}^{\dagger} T\right), F \rrbracket=\llbracket \mathcal{F} T, C_{s} F-C_{t} F \rrbracket .
$$


As $\mathcal{F} T$ is a continuous linear functional on $\mathcal{S}\left(\mathbb{R}^{2}\right)$, there is a constant $M>0$ and a continuous seminorm $p$ on $\mathcal{S}\left(\mathbb{R}^{2}\right)$ such that

$$
\left|\llbracket \mathcal{F}\left(C_{s}^{\dagger} T-C_{t}^{\dagger} T\right), F \rrbracket\right| \leq M p\left(C_{s} F-C_{t} F\right) .
$$

From the local equi-continuity property of the $C_{t}$ it now follows that the same is true for the $C_{t}^{\dagger}$. This completes the proof.

Having determined the properties of the semigroups $\left\{C_{t}: t \geq 0\right\}$ and $\left\{C_{t}^{\dagger}: t \geq 0\right\}$, we use these results to analyze $\left\{T_{t}: t \geq 0\right\}$.

Proposition 6.4. The collection $\left\{T_{t}: t \geq 0\right\}$ is a locally equicontinuous one parameter semigroup of class $C_{0}$ acting on $\mathcal{L}\left(\mathcal{S}(\mathbb{R}), \mathcal{S}^{\prime}(\mathbb{R})\right)$, with

$$
T_{t}(\boldsymbol{\Delta}[T])=\boldsymbol{\Delta}\left[C_{t}^{\dagger} T\right] .
$$

Proof. For any $T \in \mathcal{S}^{\prime}(\Pi), f, g \in \mathcal{S}(\mathbb{R})$ and $t \geq 0$,

$$
\begin{aligned}
\llbracket \widehat{T}_{t}(\Delta[T] \otimes I)(g \otimes \widetilde{\Omega}), \bar{f} \otimes \widetilde{\Omega} \rrbracket & =\llbracket(\Delta[T] \otimes I) U_{t}(g \otimes \widetilde{\Omega}), \overline{U_{t}(f \otimes \widetilde{\Omega})} \rrbracket \\
& =\llbracket \Pi\left(U_{t}(f \otimes \widetilde{\Omega}), U_{t}(g \otimes \widetilde{\Omega})\right), \boldsymbol{\Delta}[T] \rrbracket
\end{aligned}
$$

using the identification of $\mathcal{S}(\mathbb{R}) \widehat{\otimes} \mathcal{S}(\mathbb{R})$ with the dual of $\mathcal{L}\left(\mathcal{S}(\mathbb{R}), \mathcal{S}^{\prime}(\mathbb{R})\right)$.

Substituting $W[z]$ for $\boldsymbol{\Delta}[T]$ in the last expression (see equation (3.5) for $\left.x_{f, g}\right)$,

$$
\begin{aligned}
& \llbracket \Pi\left(U_{t}(f \otimes \widetilde{\Omega}), U_{t}(g \otimes \widetilde{\Omega})\right), W[z] \rrbracket=\left\langle U_{t}(f \otimes \widetilde{\Omega}), W[z](g \otimes \widetilde{\Omega})\right\rangle \\
& \quad=\left\langle f \otimes \widetilde{\Omega}, \widehat{T}_{t}(W[z] \otimes I)(g \otimes \widetilde{\Omega})\right\rangle=G_{t}(z) X_{f, g}\left(e^{-\zeta t} z\right)=\left[C_{t} X_{f, g}\right][z] .
\end{aligned}
$$

Putting the two calculations together, for all $T \in \mathcal{S}^{\prime}(\Pi), f, g \in \mathcal{S}(\mathbb{R})$ and $t \geq 0$,

$$
\begin{aligned}
\llbracket \widehat{T}_{t}(\boldsymbol{\Delta}[T] \otimes I)(g \otimes \widetilde{\Omega}), \bar{f} \otimes \widetilde{\Omega} \rrbracket & =(2 \pi)^{-1} \llbracket \mathcal{F} T, C_{t} x_{f, g} \rrbracket \\
& =(2 \pi)^{-1} \llbracket \mathcal{F} C_{t}^{\dagger} T, X_{f, g} \rrbracket
\end{aligned}
$$

Together with equation (6.4), it is now seen that $\left\{T_{t}: t \geq 0\right\}$ is a locally equicontinuous semigroup of endomorphisms of $\mathcal{L}\left(\mathcal{S}(\mathbb{R}), \mathcal{S}^{\prime}(\mathbb{R})\right)$, with equation (6.9) holding. As the quantization map is continuous, it follows that the map $t \mapsto T_{t}(X)$ is continuous from $[0, \infty)$ to $\mathcal{L}\left(\mathcal{S}(\mathbb{R}), \mathcal{S}^{\prime}(\mathbb{R})\right)$. The proof is now complete. 
Remark. With this result, we have overcome the (possible) continuity defect of the dilated family $\left\{\widehat{T}_{t}: t \geq 0\right\}$ previously noted.

We also remark that from its construction, if $X \in \mathcal{L}\left(L^{2}(\mathbb{R})\right)$, the operator $T_{t}(X)$ is equal to the restriction of $T_{t}^{(A S)}(X)$ to $\mathcal{S}(\mathbb{R})$ for any $t \geq 0$. Thus we have a non-trivial enhancement of the dynamics constructed in Alli and Sewell $[7]$.

\section{$\S 7 . \quad$ Differentiability of the Dynamical Semigroup}

From Proposition 6.4 we know that the semigroup $\left\{T_{t}: t \geq 0\right\}$ is locally equicontinuous of type $C_{0}$; we shall now prove that it is differentiable and its generator is of Lindblad type [14]. Acting on bounded operators, it will be seen to be the generator one expects for the singularly coupled oscillator. Note that there is no problem in formally identifying the putative generator: the problem is to prove that $T_{t}$ commutes with it, that $T_{t}$ is differentiable, and that the expected formula for the derivative holds on $\mathcal{L}\left(\mathcal{S}(\mathbb{R}), \mathcal{S}^{\prime}(\mathbb{R})\right)$.

For the purposes of orientation, we note that when acting on bounded operators $B$ on $L^{2}(\mathbb{R})$, the dynamical generator for this model takes the form

$$
L_{0}(B)=i \omega(N B-B N)+2 \pi g_{0}^{2} A^{+} B A-\pi g_{0}^{2}(N B+B N) .
$$

To act on mappings in $\mathcal{L}\left(\mathcal{S}(\mathbb{R}), \mathcal{S}^{\prime}(\mathbb{R})\right)$ this must be modified as follows.

Definition 7.1. By the dynamical generator we mean the continuous endomorphism $L$ of $\mathcal{L}\left(\mathcal{S}(\mathbb{R}), \mathcal{S}^{\prime}(\mathbb{R})\right)$ defined by the formula

$$
\begin{aligned}
L(X) & =i \omega\left(N^{\mathrm{t} r} X-X N\right)+2 \pi g_{0}^{2} A^{\mathrm{tr}} X A-\pi g_{0}^{2}\left(N^{\mathrm{t} r} X+X N\right), \\
& =\zeta\left(A^{\mathrm{t} r} X A-X A^{+} A\right)-\bar{\zeta}\left(A^{\mathrm{t} r}\left[A^{+}\right]^{\mathrm{tr}} X-A^{\mathrm{tr}} X A\right)
\end{aligned}
$$

for all $X \in \mathcal{L}\left(\mathcal{S}(\mathbb{R}), \mathcal{S}^{\prime}(\mathbb{R})\right)$.

In particular, for all $z \in \mathbb{C}$, it follows that

$$
L(W[z])=-i z \zeta W[z] A-i \overline{z \zeta} A^{+} W[z],
$$

which is the known action of the generator of the singularly coupled oscillator acting on the Weyl group.

A necessary condition for a one parameter semigroup to be differentiable is that it commutes with its generator. Having defined what will turn out to be the generator, let us show that the required commutation property holds. 
Proposition 7.1. For all $X \in \mathcal{L}\left(\mathcal{S}(\mathbb{R}), \mathcal{S}^{\prime}(\mathbb{R})\right), t \geq 0$,

$$
T_{t}(L(X))=L\left(T_{t}(X)\right) .
$$

Proof. Using the endomorphisms $Z_{j}$ of $\mathcal{S}\left(\mathbb{R}^{2}\right)$ and of $Z_{j}$ of $\mathcal{S}^{\prime}(\Pi)$ defined in equations (3.10a) - (3.13d), $1 \leq j \leq 4$, direct calculations yield, for any $t \geq 0$

$$
\begin{aligned}
& Z_{1} C_{t}=e^{-\zeta t} C_{t} Z_{1}, \\
& Z_{2} C_{t}=e^{-\bar{\zeta} t} C_{t} Z_{2},
\end{aligned}
$$

and so

$$
\begin{aligned}
& z_{1} C_{t}^{\dagger}=e^{\zeta t} C_{t}^{\dagger} z_{1}, \\
& z_{2} C_{t}^{\dagger}=e^{\bar{\zeta}} C_{t}^{\dagger} z_{1} .
\end{aligned}
$$

These latter results imply that, for all $X \in \mathcal{L}\left(\mathcal{S}(\mathbb{R}), \mathcal{S}^{\prime}(\mathbb{R})\right)$ and any $t \geq 0$,

$$
\begin{gathered}
T_{t}(X A)=e^{-\zeta t} T_{t}(X) A, \\
T_{t}\left(A^{\mathrm{t} r} X\right)=e^{-\bar{\zeta} t} A^{\mathrm{t} r} T_{t}(X) .
\end{gathered}
$$

To pull back the action of the generator to phase space we need the following combinations of differential operators in $z, \bar{z}$ :

$$
W_{1}=\left(Z_{2}-Z_{3}\right) Z_{1} \quad \text { and } \quad W_{2}=\left(Z_{4}-Z_{1}\right) Z_{2},
$$

which are evidently continuous endomorphisms of $\mathcal{S}(\mathbb{R})$. They commute with the $C_{t}$ : for all $F \in \mathcal{S}\left(\mathbb{R}^{2}\right)$ and all $t \geq 0$,

(7.8a) $\left[W_{1} C_{t} F\right](z)=-i z\left[Z_{1} C_{t} F\right](z)=-i z e^{-\zeta t}\left[C_{t} Z_{1} F\right](z)=\left[C_{t} W_{1} F\right](z)$,

$(7.8 \mathrm{~b})\left[W_{2} C_{t} F\right](z)=-i \bar{z}\left[Z_{2} C_{t} F\right](z)=i \bar{z} e^{-\bar{\zeta} t}\left[C_{t} Z_{2} F\right](z)=\left[C_{t} W_{2} F\right](z)$.

The $\dagger$-transpose counterparts of $W_{j}$ are $\mathcal{W}_{j}=\mathcal{F}^{-1} W_{j}^{\mathrm{tr}} \mathcal{F}, j=1$, 2. By duality, they are continuous endomorphisms of $\mathcal{S}^{\prime}(\Pi)$. Combining them with the quantization map yields the action of $L$ on phase space: for all $T \in \mathcal{S}^{\prime}(\Pi)$,

(7.9a) $\boldsymbol{\Delta}\left[\mathcal{W}_{1} T\right]=\boldsymbol{\Delta}\left[z_{1}\left(z_{2}-z_{3}\right) T\right]=A^{\text {tr }} \boldsymbol{\Delta}[T] A-\boldsymbol{\Delta}[T] A^{+} A$,

$$
\boldsymbol{\Delta}\left[\mathcal{W}_{2} T\right]=\boldsymbol{\Delta}\left[\mathcal{Z}_{2}\left(Z_{4}-Z_{1}\right) T\right]=\left[A^{+}\right]^{\operatorname{tr}} A^{\operatorname{tr}} \boldsymbol{\Delta}[T]-\left[A^{+}\right]^{\operatorname{tr}} \boldsymbol{\Delta}[T] A,
$$

so that

$$
L(\boldsymbol{\Delta}[T])=\boldsymbol{\Delta}\left[\left(\zeta \mathcal{W}_{1}-\bar{\zeta} \mathcal{W}_{2}\right) T\right]
$$


As the $W_{j}$ commute with the $C_{t}$, the $\mathcal{W}_{j}$ commute with the $C_{t}^{\dagger}$, so we can write

$$
\boldsymbol{\Delta}\left[\left(\zeta \mathcal{W}_{1}-\bar{\zeta} \mathcal{W}_{2}\right) C_{t}^{\dagger} T\right]=\Delta\left[C_{t}^{\dagger}\left(\zeta \mathcal{W}_{1}-\bar{\zeta} \mathcal{W}_{2}\right) T\right]
$$

Now on the one hand,

$$
\boldsymbol{\Delta}\left[\left(\zeta \mathcal{W}_{1}-\bar{\zeta} \mathcal{W}_{2}\right) C_{t}^{\dagger} T\right]=L\left(\boldsymbol{\Delta}\left[C_{t}^{\dagger} T\right]\right)=L T_{t}(\boldsymbol{\Delta}[T])
$$

and on the other,

$$
\boldsymbol{\Delta}\left[C_{t}^{\dagger}\left(\zeta \mathcal{W}_{1}-\bar{\zeta} \mathcal{W}_{2}\right) T\right]=T_{t}\left(\boldsymbol{\Delta}\left[\left(\zeta \mathcal{W}_{1}-\bar{\zeta} \mathcal{W}_{2}\right) T\right]\right)=T_{t} L(\boldsymbol{\Delta}[T])
$$

Thus, for all $t \geq 0, L$ commutes with $T_{t}$, completing the proof.

The next step in constructing the derivative of $T_{t}$ is to take the derivative of $C_{t}\left(X_{f, g}\right)$. This lemma is the key to the solution.

Lemma 7.1. For all $f, g \in \mathcal{S}(\mathbb{R}), z \in \mathbb{C}$ and $t \geq 0$,

$$
\partial_{t}\left[C_{t} X_{f, g}\right](z)=2 \pi g_{0}^{2}\left[C_{t} X_{A f, A g}\right](z)-\bar{\zeta}\left[C_{t} X_{N f, g}\right](z)-\zeta\left[C_{t} X_{f, N g}\right](z)
$$

Proof. We note that, for all $f, g \in \mathcal{S}(\mathbb{R}), z \in \mathbb{C}$ and $t \geq 0$,

$$
\left[C_{t} X_{f, g}\right][z]=G_{t}(z) X_{f, g}\left(e^{-\zeta t} z\right)=e^{-|z|^{2} / 2}\left\langle f, e^{i e^{-\zeta t} z A^{+}} e^{i e^{-\zeta t} z A} g\right\rangle .
$$

This may be differentiated with respect to $t$, yielding

$$
\begin{aligned}
\partial_{t}\left[C_{t} x_{f, g}\right](z)= & -i \overline{z \zeta} e^{-\bar{\zeta} t}\left[C_{t} x_{A f, g}(z)-i z \zeta e^{-\zeta t}\left[C_{t} x_{f, A g}(z)\right.\right. \\
= & -i \overline{z \zeta} e^{-\bar{\zeta} t}\left\langle A f, T_{t}(W[z]) g\right\rangle-i z \zeta e^{-\zeta t}\left\langle f, T_{t}(W[z]) A g\right\rangle \\
= & -i \overline{z \zeta}\left\langle f, T_{t}\left(A^{+} W[z]\right) g\right\rangle-i z \zeta\left\langle f, T_{t}(W[z] A) g\right\rangle \\
= & \left\langle f, T_{t}(L\{W[z]\}) g\right\rangle=\left\langle f, L\left(T_{t}\{W[z]\}\right) g\right\rangle \\
= & 2 \pi g_{0}^{2}\left\langle A f, T_{t}\{W[z]\} A g\right\rangle-\bar{\zeta}\left\langle N f, T_{t}\{W[z]\} g\right\rangle \\
& -\zeta\left\langle f, T_{t}\{W[z]\} N g\right\rangle
\end{aligned}
$$

for all $f, g \in \mathcal{S}(\mathbb{R}), z \in \mathbb{C}$ and $s, t \geq 0$, from which the result follows.

Our next task is to find a continuity estimate for $C_{s} x_{f, g}-C_{t} x_{f, g}$ which will lead to proof of differentiability of $T_{t}$. We already have an estimate, equation (6.6), but it has the disadvantage that we have no control on the indices on the right hand side. As the inequality we use will have to be iterated, the bound we have is not quite adequate. At the expense of introducing a certain bounded subset of $\mathcal{S}\left(\mathbb{R}^{2}\right)$, the following estimate overcomes this difficulty. 
Lemma 7.2. Let $\tau>0$. For $0<s, t \leq \tau$, and $j, k, m, n \in \mathbb{Z}^{+}$,

$$
\begin{aligned}
& Z_{j k m n}\left(C_{s} x_{f, g}-C_{t} X_{f, g}\right) \\
& \quad \leq\left(2 \pi g_{0}^{2}+2|\zeta|\right)|s-t| \sup \left\{Z_{j k m n}(F): F \in \mathcal{B}(f, g, \tau)\right\}
\end{aligned}
$$

where

$$
\mathcal{B}(f, g, \tau)=\bigcup_{0 \leq u \leq \tau}\left\{C_{u} X_{A f}, A g, C_{u} X_{N f, g}, C_{u} X_{f, N g}\right\}
$$

is a bounded subset of $\mathcal{S}\left(\mathbb{R}^{2}\right)$ for any $f, g \in \mathcal{S}(\mathbb{R})$ and $\tau \geq 0$.

Proof. The boundedness of $\mathcal{B}(f, g, \tau)$ follows because the family $\left\{C_{t}: t \geq 0\right\}$ is a locally equicontinuous set of endomorphisms of $\mathcal{S}\left(\mathbb{R}^{2}\right)$.

The starting point for equation (7.12) is to integrate the derivative of $C_{t} X_{f, g}$ just obtained: for all $f, g \in \mathcal{S}(\mathbb{R}), z \in \mathbb{C}$ and $s, t \geq 0$,

$$
\begin{gathered}
C_{s} x_{f, g}(z)-C_{t} x_{f, g}(z)=\int_{t}^{s}\left\{2 \pi g_{0}^{2}\left[C_{u} x_{A f, A g}\right](z)-\bar{\zeta}\left[C_{u} x_{N f, g}\right](z)\right. \\
\left.-\zeta\left[C_{u} x_{f, N g}\right](z)\right\} d u
\end{gathered}
$$

The asserted bound requires us to show that $C_{s} x_{f, g}-C_{t} x_{f, g} \in \mathcal{S}\left(\mathbb{R}^{2}\right)$, and to do that we must pair it with arbitrary $T \in \mathcal{S}^{\prime}\left(\mathbb{R}^{2}\right)$. However, we cannot just commute the pairing with the integration, and so will proceed indirectly, using the fact that $\mathcal{S}\left(\mathbb{R}^{2}\right)$ is reflexive.

We define the expression that would result from placing the pairing inside the integrand: for all $f, g \in \mathcal{S}(\mathbb{R}), z \in \mathbb{C}$ and $s, t \geq 0$, let

$$
\begin{aligned}
J_{f g ; s t}(T)=\int_{t}^{s} & \left\{2 \pi g_{0}^{2} \llbracket T, C_{u} x_{A f, A g} \rrbracket(z)-\bar{\zeta} \llbracket T, C_{u} x_{N f, g} \rrbracket(z)\right. \\
& \left.-\zeta \llbracket T, C_{u} x_{f, N g} \rrbracket(z)\right\} d u .
\end{aligned}
$$

This exists for all $T \in \mathcal{S}^{\prime}\left(\mathbb{R}^{2}\right)$, and defines a linear functional on $\mathcal{S}^{\prime}\left(\mathbb{R}^{2}\right)$,

$$
\left|J_{f g ; s t}(T)\right| \leq\left(2 \pi g_{0}^{2}+2|\zeta|\right)|s-t| \sup \{|\llbracket T, F \rrbracket|: F \in \mathcal{B}(f, g, \tau)\}
$$

for all $f, g \in \mathcal{S}(\mathbb{R}), s, t \in[0, \tau]$ and $T \in \mathcal{S}^{\prime}\left(\mathbb{R}^{2}\right)$.

Hence

$$
J_{f g ; s t}=\llbracket T, I_{f, g}(s, t) \rrbracket
$$


defines an element $I_{f, g}(s, t) \in \mathcal{S}^{\prime \prime}\left(\mathbb{R}^{2}\right) \cong \mathcal{S}\left(\mathbb{R}^{2}\right)$, and equation (7.16) implies that

$$
\left|\llbracket T, I_{f, g}(s, t) \rrbracket\right| \leq\left(2 \pi g_{0}^{2}+2|\zeta|\right)|s-t| \sup \{|\llbracket T, F \rrbracket|: F \in \mathcal{B}(f, g, \tau)\}
$$

for any $f, g \in \mathcal{S}(\mathbb{R}), s, t \in[0, \tau]$ and $T \in \mathcal{S}^{\prime}\left(\mathbb{R}^{2}\right)$.

Applying the seminorm $Z_{j k m n}$ to this,

$$
\begin{aligned}
& Z_{j k m n}\left(I_{f, g}(s, t)\right) \\
& \quad \leq\left(2 \pi g_{0}^{2}+2|\zeta|\right)|s-t| \sup \left\{Z_{j k m n}(F): F \in \mathcal{B}(f, g, \tau)\right\} .
\end{aligned}
$$

The connection with $C_{s} x_{f, g}-C_{t} x_{f, g}$ now follows by substituting the Dirac delta distribution $\delta_{z}$ for $T$ : for all $f, g \in \mathcal{S}(\mathbb{R}), s, t \geq 0$ and $z \in \mathbb{C}$,

$$
\llbracket \delta_{z}, C_{s} x_{f, g}-C_{t} x_{f, g}-I_{f, g}(s, t) \rrbracket=0,
$$

so we are now able to say that, for all $f, g \in \mathcal{S}(\mathbb{R})$ and $s, t \geq 0$,

$$
C_{s} X_{f, g}-C_{t} X_{f, g}=I_{f, g}(s, t) .
$$

Therefore, with $0<s, t \leq \tau$ and $j, k, m, n \in \mathbb{Z}^{+}$,

$$
Z_{j k m n}\left(C_{s} X_{f, g}-C_{t} X_{f, g}\right)=Z_{j k m n}\left(I_{f, g}(s, t)\right) .
$$

Equation (7.18) now completes the proof.

We now have the bound that will allow us to prove differentiability of $T_{t}$.

Proposition 7.2. The semigroup $t \mapsto T_{t}(X)$ is differentiable from $[0, \infty)$ to $\mathcal{L}\left(\mathcal{S}(\mathbb{R}), \mathcal{S}^{\prime}(\mathbb{R})\right)$, with

$$
\frac{d}{d t} T_{t}(X)=L\left(T_{t}(X)\right)=T_{t}(L(X)), \quad X \in \mathcal{L}\left(\mathcal{S}(\mathbb{R}), \mathcal{S}^{\prime}(\mathbb{R})\right), t \geq 0
$$

Proof. Let $X=\boldsymbol{\Delta}[T]$ be an arbitrary element of $\mathcal{L}\left(\mathcal{S}(\mathbb{R}), \mathcal{S}^{\prime}(\mathbb{R})\right)$. Then for $f, g \in \mathcal{S}(\mathbb{R})$ and $s, t \geq 0$,

$$
\begin{aligned}
& \llbracket T_{s}(X) g, \bar{f} \rrbracket-\llbracket T_{t}(X) g, \bar{f} \rrbracket \\
& =(2 \pi)^{-1} \llbracket \mathcal{F} T, C_{s} X_{f, g}-C_{t} X_{f, g} \rrbracket=(2 \pi)^{-1} \llbracket \mathcal{F} T, I_{f, g}(s, t) \rrbracket \\
& =\int_{t}^{s}\left\{2 \pi g_{0}^{2} \llbracket T_{u}(X) A g, \overline{A f} \rrbracket-\bar{\zeta} \llbracket T_{u}(X) g \overline{N f} \rrbracket-\zeta \llbracket T_{u}(X) N g, \bar{f} \rrbracket\right\} d u .
\end{aligned}
$$


Therefore, using equation (7.2b),

$$
\begin{aligned}
& \llbracket T_{s}(X) g, \bar{f} \rrbracket-\llbracket T_{t}(X) g, \bar{f} \rrbracket-(s-t) \llbracket L\left(T_{t}(X)\right) g, \bar{f} \rrbracket \\
&=\int_{t}^{s}\left\{2 \pi g_{0}^{2}\left\{\llbracket T_{u}(X) A g, \overline{A f} \rrbracket-\llbracket T_{t}(X) A g, \overline{A f} \rrbracket\right\}\right. \\
&- \bar{\zeta}\left\{\llbracket T_{u}(X) g, \overline{N f} \rrbracket-\llbracket T_{t}(X) g, \overline{N f} \rrbracket\right\} \\
&\left.-\zeta\left\{\llbracket T_{u}(X) N g, \bar{f} \rrbracket-\llbracket T_{t}(X) N g, \bar{f} \rrbracket\right\}\right\} d u
\end{aligned}
$$

We shall now show that this is of order $|s-t|^{2}$ weakly. Writing the integral in the form

$$
\int_{t}^{s}\{I+I I+I I I\} d u
$$

in an obvious notation. Estimating $I, I I$ and $I I I$ in turn:

$$
\begin{gathered}
|I| \leq 4 \pi g_{0}^{2}\left(\pi g_{0}^{2}+|\zeta|\right)|u-t| \sup \left\{Z_{j k m n}(F): F \in \mathcal{B}(A f, A g, \tau)\right\} \\
|I I| \leq 2|\zeta|\left(\pi g_{0}^{2}+|\zeta|\right)|u-t| \sup \left\{Z_{j k m n}(F): F \in \mathcal{B}(N f, g, \tau)\right\} \\
|I I I| \leq 2|\zeta|\left(\pi g_{0}^{2}+|\zeta|\right)|u-t| \sup \left\{Z_{j k m n}(F): F \in \mathcal{B}(f, N g, \tau)\right\} .
\end{gathered}
$$

Writing

$$
\mathcal{D}(f, g, \tau)=\mathcal{B}(A f, A g, \tau) \bigcup \mathcal{B}(N f, g, \tau) \bigcup \mathcal{B}(f, N g, \tau),
$$

we have the bound

$$
\begin{aligned}
& \left|\llbracket T_{s}(X) g, \bar{f} \rrbracket-\llbracket T_{t}(X) g, \bar{f} \rrbracket-(s-t) \llbracket L\left(T_{t}(X)\right) g, \bar{f} \rrbracket\right| \\
& \quad \leq \frac{K}{\pi}\left(\pi g_{0}^{2}+|\zeta|\right)|s-t|^{2} \sup \left\{Z_{j k m n}(F): F \in \mathcal{D}(f, g, \tau)\right\},
\end{aligned}
$$

where the positive constant $K$ depends on $T, j, k, m, n$ and is chosen so that, for all $F \in \mathcal{S}\left(\mathbb{R}^{2}\right)$,

$$
|\llbracket \mathcal{F} T, F \rrbracket| \leq K Z_{j k m n}(F) .
$$

The construction of the bounded set $\mathcal{D}(f, g, \tau)$, depending on the local equicontinuity of $\left\{C_{t}: t \geq 0\right\}$, is such that there exists a positive constant $K^{\prime}$ (depending on $K$ ) and an integer $r \geq 0$ such that, for all $f, g \in \mathcal{S}(\mathbb{R})$ and $s$, $t \in[0, \tau]$,

$$
\begin{aligned}
& \left|\llbracket T_{s}(X) g, \bar{f} \rrbracket-\llbracket T_{t}(X) g, \bar{f} \rrbracket-(s-t) \llbracket L\left(T_{t}(X)\right) g, \bar{f} \rrbracket\right| \\
& \quad \leq K^{\prime}|s-t|^{2} \widehat{q}_{r}(f) \widehat{q}_{r}(g) .
\end{aligned}
$$


It follows immediately that, for all $F \in \mathcal{S}\left(\mathbb{R}^{2}\right)$,

$\left|\llbracket F, T_{s}(X) \rrbracket-\llbracket F, T_{t}(X) \rrbracket-(s-t) \llbracket F, L\left(T_{t}(X)\right) \rrbracket\right| \leq K^{\prime}|s-t|^{2} \widehat{q}_{r} \widehat{\otimes} \widehat{q}_{r}(F)$, from which differentiability is immediate.

\section{Appendix}

A number of families of seminorms are used in this paper, and we have listed them here. The topology on $\mathcal{S}(\mathbb{R})$ is defined by the family $\left\{\widehat{q}_{r}: r \in \mathbb{Z}^{+}\right\}$ of seminorms, where

$$
\widehat{q}_{s}(f)=\max _{0 \leq r \leq s} \max \left\{\left\|A^{\sharp_{1}} \cdots A^{\sharp_{r}} f\right\|: \sharp_{1}, \ldots, \sharp_{r}\right\} .
$$

Here $A^{\sharp}$ is either $A$ or $A^{+}$, the lowering and raising operator, respectively, and the inner maximum is taken over the $2^{r}$ possible choices.

An equivalent family is $\left\{\tilde{p}_{r}: r \in \mathbb{Z}^{+}\right\}$, where

$$
\tilde{p}_{r}(f)=\left\|(N+1)^{r / 2} f\right\|, \quad f \in \mathcal{S}(\mathbb{R}), r \in \mathbb{Z}^{+},
$$

where $N=A^{+} A$ is the number operator.

Complex conjugation combines with the ladder operators as $A \bar{f}=\overline{A^{+} f}$ and $A^{+} \bar{f}=\overline{A f}$, so for all $r \in \mathbb{Z}^{+}, f \in \mathcal{S}(\mathbb{R})$,

$$
\widehat{q}_{r}(\bar{f})=\widehat{q}_{r}(f) \text {. }
$$

The topology on $\mathcal{S}\left(\mathbb{R}^{2}\right)$ is determined by the seminorm family $\left\{Z_{j k m n}: j\right.$, $\left.k, m, n \in \mathbb{Z}^{+}\right\}$, where

$$
Z_{j k m n}(F)=\sup _{z \in \mathbb{C}}\left|z^{j} \bar{z}^{k} \partial^{m} \bar{\partial}^{n} F(z)\right| .
$$

For the locally convex topology on the 'universe' space $\widehat{\mathcal{Q}}^{(0)}$ introduced in equation (4.3) we have the seminorms $\left\{q_{r s}: r, s \in \mathbb{Z}^{+}\right\}$: for any $r, s \in \mathbb{Z}^{+}$, $\widehat{\Phi} \in \widehat{\mathcal{Q}}^{(0)}$,

$$
\text { (A.5a) } \begin{aligned}
& q_{r s}(\widehat{\Phi}) \\
= & \sup \left\{\left\|A^{\sharp_{1}} \cdots A^{\sharp_{r}} \otimes \widetilde{w}\left(f_{1}\right) \cdots \widetilde{w}\left(f_{s}\right) \widehat{\Phi}\right\|: \sharp_{1}, \ldots, \sharp_{r}, f_{1}, \ldots, f_{s} \in \mathfrak{b}\right\}
\end{aligned}
$$

Two equivalent families are (in both cases $r, s \in \mathbb{Z}^{+}$):

$$
p_{r s}(\widehat{\Phi})=\max _{0 \leq u \leq r, 0 \leq v \leq s} \sup \left\{\left\|A^{r} \otimes \widetilde{w}\left(f_{1}\right) \cdots \widetilde{w}\left(f_{s}\right) \widehat{\Phi}\right\|: f_{1}, \ldots, f_{s} \in \mathfrak{b}\right\}
$$

$$
\tilde{p}_{r s}(\widehat{\Phi})=\sup \left\{\left\|(N+1)^{r} \otimes \widetilde{w}\left(f_{1}\right) \cdots \widetilde{w}\left(f_{s}\right) \widehat{\Phi}\right\|: f_{1}, \ldots, f_{s} \in \mathfrak{b}\right\}
$$




\section{References}

[1] Dubin, D. A. and Hennings, M. A., Quantum Mechanics, Algebras and Distributions, Pitman/Longman (Harlow, Essex), 1990.

[2] Roberts, J. E., Rigged Hilbert Spaces in quantum Mechanics, Comm. Math. Phys., 3 (1966), 98-109.

[3] The Dirac bra and ket formalism, J. Math. Phys., 7 (1966), 1097-1104.

[4] Dubin, D. A., Hennings, M. A. and Smith, T. B., Mathematical Aspects of Weyl Quantization and Phase, World Scientific Press (Singapore), 2000.

[5] Folland, G. B., Harmonic Analysis in Phase Space, Princeton University Press, 1989.

[6] Weyl, H., Group Theory and Quantum Mechanics, Methuen (London), 1931; reprinted Dover Publications (New York), 1950.

[7] Alli, G. and Sewell, G. L., New methods and structures in the theory of the multimode Dicke laser model, J. Math. Phys., 36 (1995), 5598-5626.

[8] Hudson, R. L. and Parthasaraty, K. R., Quantum Ito's formula and stochastic evolutions, Comm. Math. Phys., 93 (1984), 301-323.

[9] Hepp, K. and Lieb, E., Phase transitions in reservoir driven open systems with application to lasers and super-conductors, Helv. Phys. Acta, 46 (1973), 573-603.

[10] Jarchow, H., Locally Convex Spaces, Teubner (Stuttgart), 1981.

[11] Schaeffer, H. H., Topological Vector Spaces, New York: Springer (New York, Heidelberg, Berlin), 1971

[12] Trevès, F., Topological Vector Spaces, Distributions and Kernels, Academic Press (New York), 1967.

[13] Yosida, K., Functional Analysis, Springer (Berlin, Heidelberg, New York), 1980.

[14] Lindblad, G., On the generators of quantum dynamical semigroups, Comm. Math. Phys., 48 (1976), 119-130. 